\title{
Design of a Small-Scale Experimental Model of the International Space Station Crew Quarters for a PIV Flow Field Study
}

\author{
Matei-Razvan Georgescu ${ }^{1,2}$, Ilinca Nastase ${ }^{1}$, Amina Meslem², Mihnea Sandu ${ }^{1}$, Florin Bode ${ }^{1,3,}$ \\ ${ }^{1}$ Technical University of Civil Engineering Bucharest, Romania. \\ ${ }^{2}$ Université de Rennes 1, France. \\ ${ }^{3}$ Technical University of Cluj-Napoca, Romania.
}

\begin{abstract}
An attempt at improving the ventilation solution for the crew quarters aboard the International Space Station requires a thorough understanding of the flow dynamics in a microgravity environment. An experimental study is required in order to validate the numerical models. As part of this process, a small-scale model was proposed for a detailed study of the velocity field. PIV measurements in water offer high quality results and were chosen for the subject. Following certain similitude criteria, an equivalence can be found between the results of these measurements and the real ventilation scenario. This paper describes the development process of this small-scale model as well as its performance in the initial test runs. Details regarding the advantages and weaknesses of this first model are the core of the paper, with the intention of aiding researchers in their design of similar models. The conclusion presents future steps and proposed improvements to the model.
\end{abstract}

\section{Introduction}

Ventilation systems on the International Space Station (ISS) are of paramount importance for the well-being of the astronauts on board. Good airflow is a requirement in confined work spaces and it is essential in the context of outer space missions. In the absence of gravity, natural convection is not present due to density difference between hot and cold air not being a factor. This leads to issues regarding air stagnation, particularly near the face of a stationary person. The exhaled air, containing a high concentration of $\mathrm{CO}_{2}$ accumulates around an astronaut's head [1] and can cause headaches and other $\mathrm{CO}_{2}$ intoxication effects, presenting a risk of asphyxiation in the worst of cases [2]. The general ventilation system aboard the ISS is adequate for the large majority of these situations, however one case where this does not entirely apply is the sleeping quarters of the astronauts.

The crew quarters (CQ) are small cabins where the astronauts sleep. There are multiple reports [3][4][5] stating that astronauts are waking up with symptoms of $\mathrm{CO}_{2}$ intoxication, indicating a higher concentration inside the crew quarters than expected.

As part of a research endeavor to find better ventilation solutions for the crew quarters on the ISS, a numerical study is required in order to study the ventilation solutions in micro-gravity. Numerical models require experimental validation which in this case is to be done with a small-scale experimental model of the CQ which enables Particle Image Velocimetry (PIV) measurements of the flow fields with high spatial resolution. The equipment used is a Dantec PIV system, composed of a high sensitivity Flow Sense 4M camera with 4x106 pixels resolution and a Dual Power 200mJ with a wave length of $532 \mathrm{~nm}$. The PIV measurements are realized in water. The equivalence between the flow in the small-scale model in water and the full-scale model with air is calculated according to similitude criteria, namely similitude of the Reynolds number [6].

This document concerns the design process and preliminary results of this small-scale experimental model from the standpoint of technical solutions applicable to the design of water PIV studies in small-scale experimental models.

\section{Experimental Model Design}

The small-scale model was scaled to $1 / 4^{\text {th }}$ of the original size. For a PIV study, at least two transparent surfaces are required, one through which the laser itself can illuminate the plane of study and another through which the specialized high-resolution camera will visualize the flow field. 
The small-scale model was created out of transparent acrylic. The hydraulic circuits behind the introduction and extraction diffusers were not reproduced exactly as they are found in the full-scale model since the more complex design would be difficult to realize. It was also deemed that the only area of interest is the interior of the model, hence if the flow field distribution at the diffuser could be reproduced in the small-scale model, the ventilation circuit itself would be of little interest. A design schema of the experimental setup can be seen in Fig. 1, while Fig. 2 shows the small-scale model from behind the Flow Sense camera.



Fig. 1. Design schema of the experimental setup.

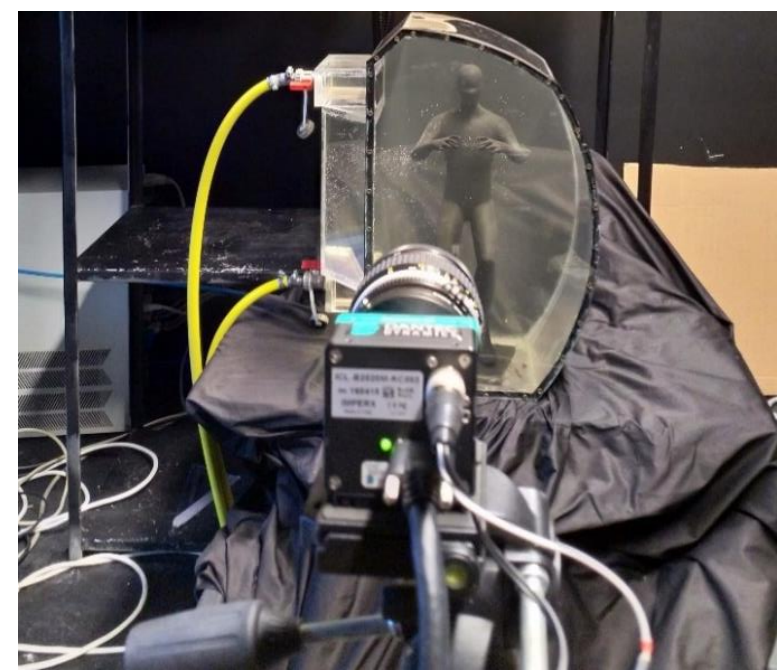

Fig. 2. Small scale model setup.

A parallelepipedal plenum chamber was designed before the introduction grid supplied with water through a 1-inch pipe. The purpose of this plenum chamber is the stabilization of the flow before the diffuser. The streamwise length of this region is about 3-4 diameters. A similar region was designed for the extraction region with a pipe of the same diameter. The interior of the model is accessible via the front panel (see Fig. 3) which is fixed with screws and waterproofed via a gasket. Two types of gaskets were available, one made out of rubber, the other out of KlingerTM. The screws themselves are inserted directly into the acrylic of both the removeable wall and the rest of the basin.

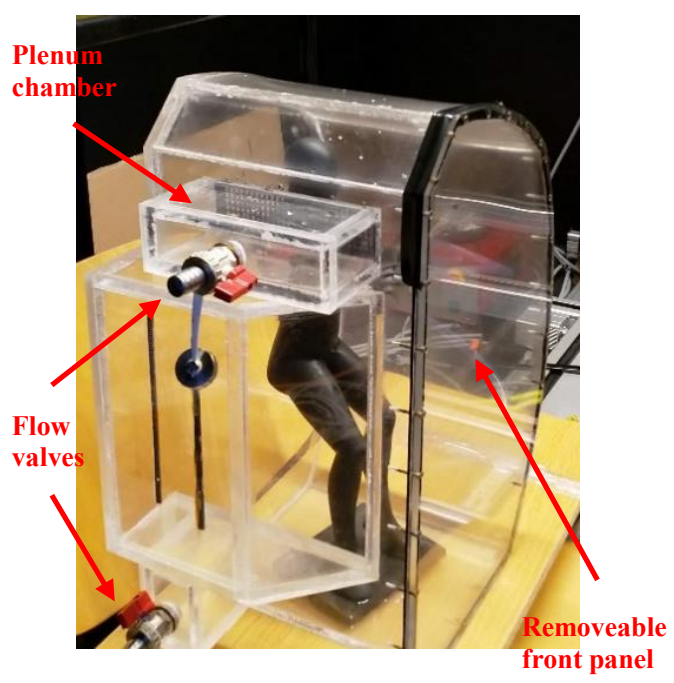

Fig. 3. Small scale model water supply

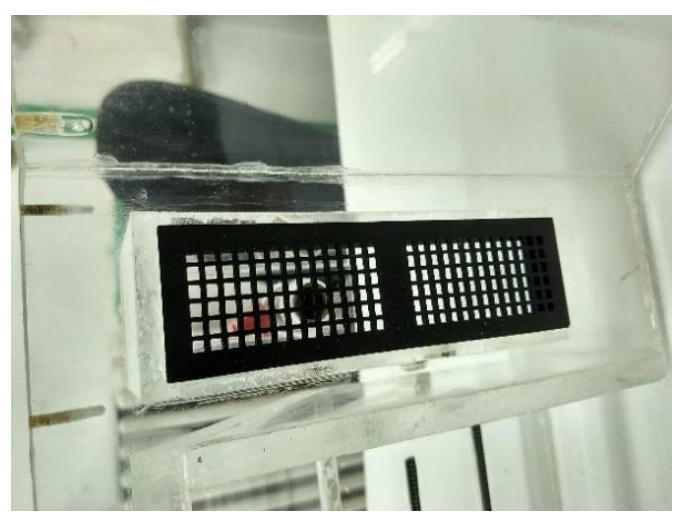

Fig. 4. 3D printed diffuser.

Access inside the model was necessary for the inlet diffuser and for the small-scale human model of an astronaut that was decided to be put inside in order to test the influence of the human body presence on the flow distribution.

It was decided that the inlet diffuser and the smallscale human model placed inside the CQ model would be designed with the help of a 3D printer. The diffuser was designed as a $1 / 4^{\text {th }}$ scale replica of the one placed in the full-scale model. The human model was set in a position that represents the natural body posture in zero gravity [2] (Fig. 4). The dimensions of the human body model were chosen to fit the scale of our human body numerical models and keeping the proportions of one thermal manikin that was developed in our team [7][8].

Fixing the human model in place was done with the help of a small acrylic plate to which the human's feet were connected with screws. The acrylic plate was then glued to the bottom of the acrylic basin. Another small acrylic cylinder was glued to the back of the human model fixed with hot glue to the plastic model and with acrylic glue to the back wall. This last fixture was done in order to prevent the swaying of the model during water flow. It 
was judged that the influence of the small $0,5 \mathrm{~cm}$ diameter cylinder on the flow field would be minimal as well as not hindering the camera due to being situated on the other side of the human model from the camera's point of view. In addition, having the human model stay in one place enabled the fixing of measuring planes for the laser.
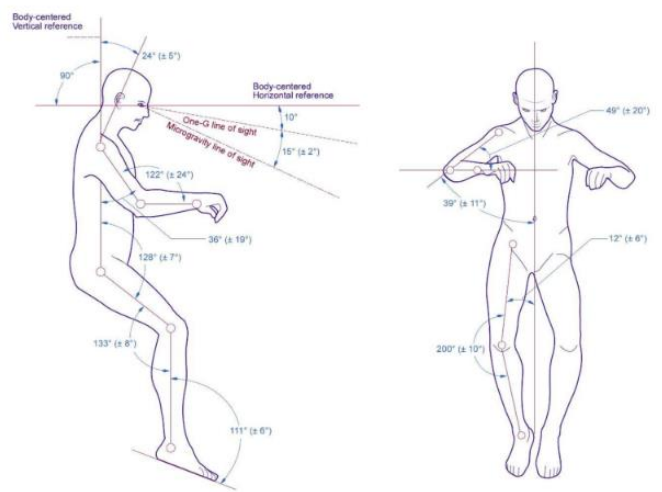

Fig. 5. Neutral human body posture in micro-gravity [2].



Fig. 6. 3D printed human, fixed inside the small-scale model

The small-scale diffuser was fixed in its place with silicone. For waterproofing the basin either of the gaskets can be used to seal the space between the removable basin wall and the rest of the model. No other adjustments were made to the small-scale model's structure.

The water was supplied by a nearby pump connected to a reservoir. The circuit was built from a series of hoses tightened with hose clamps. This was done to ensure easy switching between admission and evacuation supply pipes during the initial filling of the small-scale model. Three types of reflective tracers were used for the visualization of the flow.

\section{Experimental Setup}

\subsection{First test}

The first experimental test was done as a reference case, with no small-scale human model inside and without the diffuser, using the Klingerit ${ }^{\mathrm{TM}}$ gasket for sealing and Iriodin ${ }^{\circledR} 100$ Silver Pearl particles for flow visualization. These particles are polydisperse on a large range of diameters from 10 to $100 \mu \mathrm{m}$.

From the onset, the complex geometry of the model caused problems in terms of air pocket appearance. First of all, the model initially lacked an air evacuation due to a miscommunication with the producers. This enabled large pockets of air to form in the top side of the model and one side in a protruding region of the model. The air was evacuated for this first test by turning the model to one side so the air could escape through the top supply duct. This method was however imperfect due to the fact that initially in order for the air to escape at all the model had to be filled with water from the bottom up, which resulted in a couple of air bubbles becoming trapped inside the model upon switching the supply ducts to their proper places.

The Iriodin ${ }^{\circledR} 100$ Silver Pearl particles were introduced in the preliminary PIV measurements which had to be ceased due to leakage problems.

The Klingerit ${ }^{\mathrm{TM}}$ seal must be tightened very firmly in order to function as intended. The initial sealing process went well with the exception of a few screw holes in the acrylic wall which had a deteriorated fillet. After filling the model with water, the pressure applied on the removeable wall due to the weight of the water caused a weakening in the seal. This phenomenon was especially prevalent in the inferior region of the model where the pressure was higher. All of the above resulted in the appearance of leakages which only worsened over time causing the above-mentioned cessation of the measurement process. Upon opening the CQ model to assess the situation, several things were observed. First of all, the weakening of the seal had a secondary effect, that of the weakening of the screws used to seal the model. The force applied to the wall by the water pressure deteriorated the fillet of the screws inside the acrylic wall thus worsening an already existing problem. Coupling this with the fact that Klingerit ${ }^{\mathrm{TM}}$ requires a very tight seal was the cause of all the leakages.

The second issue was with the Iriodin ${ }^{\circledR} 100$ Silver Pearl powder which was found to have stuck to the walls of the model, generating a matte effect which would have hampered both camera and laser measurements. While this issue was expected to a degree, the unexpected part was the difficulty in cleaning the model of this powder. Several cleaning passes had to be made in order for the 
acrylic to regain its transparency resulting in a fair amount of lost time.

\subsection{Second test}

Building upon the results of the previous test the following adjustments were made. The Klingerit ${ }^{\mathrm{TM}}$ seal was replaced with a rubber one and the powder used was changed from S-HGS, Silver Coated Hollow Glass Spheres of $10 \mu \mathrm{m}$ to PMMA-RhB-10, polydisperse fluorescent polymer particles with diameters in the range $1-20 \mu \mathrm{m}$ in the hopes of lessening the time required to clean the model. The $3 \mathrm{D}$ printed human and the diffuser were also introduced in this second test in order to test the resistance of the silicone fit of the diffuser and the bottom of the human model as well as the fastening system on the back of the human model with the aid of hot glue to the model itself and to the rear acrylic wall. The possibility of drilling a hole in the top side of the model was discussed but was rejected at the time as the risk of cracks appearing in the plane through which the laser had to pass through was considered too great.

The rubber gasket held well with only very minor leaks in a region where the screws were weakly fixed due to the deterioration of the acrylic fillet. A decision was made to increase the flow rate inside the model, however with the gradual increase in flow rate the rear basin wall tore open at its seam rendering the model unusable. In addition, the silicone fitting of the printed diffuser weakened as did the connection between the human model and the rear wall. The rubber gasket however proved to be a better solution presenting moderate leaks in the instants before the basin seam cracked.

Before the third test could begin, the model seam was repaired using ACRIFIX acrylic glue. The top of the model presented several scratches as a result of the reparations and thus would have to be polished until transparent. During the reparations a hole was also drilled in the top of the model for the evacuation of air. The small cracks generated by drilling the hole were repaired during the polishing process and the hole itself being as far as possible from the measuring plane was plugged with silicone. In this silicone plug a syringe needle was introduced in order to permit the extraction of air by natural and forced means if necessary.

The silicone fit of the diffuser was remade in order to avoid any weakened points. The base of the human model held well, although the fitting which glued to back of the model to the rear acrylic wall came loose. This was due to the hot glue's inefficiency between two acrylic surfaces. It was replaced by ACRIFIX opting for a more permanent solution by fusing together the acrylic support to the rear wall. Due to ACRIFIX's properties it was also introduced in small quantities in the weakened screw fillets just before the screws themselves were inserted. The idea would be that the ACRIFIX would fuse with the surrounding acrylic and around the screw forming a very tight fit which would reduce leakages.

\subsection{Third test}

During the third test all of the hypotheses presented above proved to be accurate, the air gathered in the top side of the basin was easily evacuated, the diffuser and the human model remained firmly in place and rubber gasket leakages were at an absolute minimum. The flow rate was increased to the point where the leakages near the gasket were manageable and the resulting flow rate was measured at around $7 \mathrm{l} / \mathrm{min}$.

For this test the PMMA-RhB-10 was used with a special fluorescent filter applied to the camera. No other notable issues arose from this last setup and the results obtained are deemed the most reliable.

Measurements were made at different planes perpendicular to the diffuser as shown below in Fig. 7. The three measurement sections named Section 1, 2 and 3 (S1, S2, S3) have been chosen as the measurement planes. $\mathrm{S} 1$ was chosen because it is in the middle of the diffuser and provides information about the flow in front of the filled middle section. S2 aims to provide a view of the jet flow near the middle filled, and finally S3 is positioned near the middle of the left side of the grid, corresponding to a median plane through the jet on the left side of the grid. The aim of the measurements was to have a clear picture of the flow field in planes perpendicular to the diffuser and passing near the head region of the human model.



Fig. 7. Three measurement planes: Section 1 (orange), Section 2 (red) and Section 3 (green)

\section{Results and discussion}

The primary criteria to be considered is the water velocity at the exit of the diffuser. Knowing the flow rate and the effective open surface of the diffuser, it is possible to determine an average velocity through the grid, offering an estimate of the expected order of magnitude. The open surface is calculated by adding up the surfaces of the 156 rectangular openings of the diffuser which can be seen in Fig. 7. For a flow rate of $71 / \mathrm{min}$ and an open surface of $24.96 \mathrm{~cm}^{2}$ the average velocity should be approximately $0.05 \mathrm{~m} / \mathrm{s}$.

Figure 8 represents the median measurement plane situated at the middle of the diffuser (S1 in Fig. 7), where there is a filled area. Naturally, at this position velocity values are small near the diffuser exit and they increase when the elementary jets adjacent to the middle section merge. 


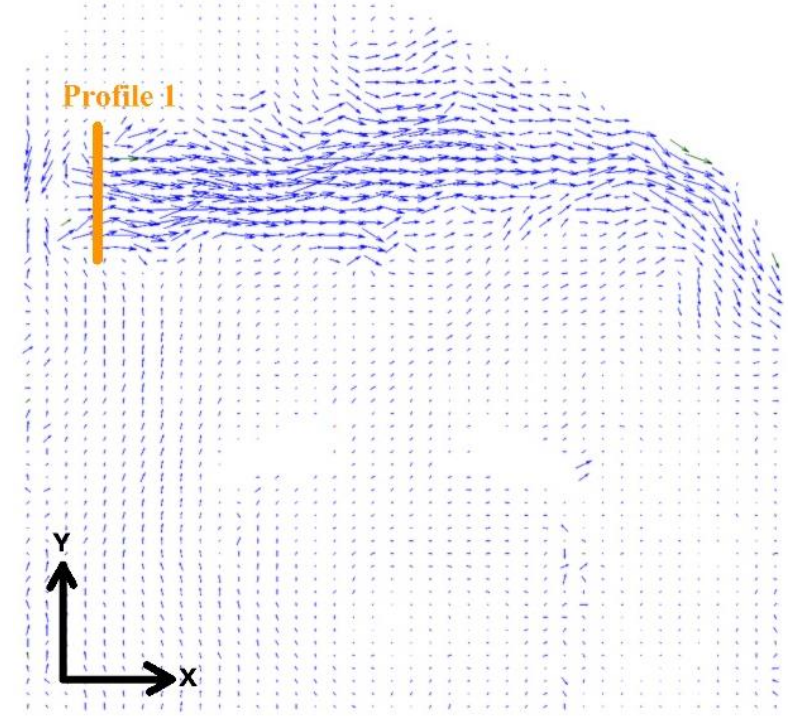

Fig. 8. Median plane velocity vectors (S1), velocity profile marked Profile 1 , situated $3 \mathrm{~cm}$ in front of the diffuser.

Figure 8 presents the velocity profiles in the plane corresponding to section 2 in Fig.7. The flow direction can clearly be seen. White regions inside the flow field as well as around it are due to value blanking of the walls of the small-scale model as well as the human model.



Fig. 9. Velocity vectors at measurements section S2, velocity profile marked Profile 2, situated $3 \mathrm{~cm}$ in front of the diffuser.

Figure 9 presents the velocity profiles in the plane corresponding to section S3 in Fig.7. The flow field in front of the grid for this measurement section appears to be chaotic, not presenting the clear pattern of Figure 8 , while at times vectors can be seen directed towards the grid indicating an aspiration effect.

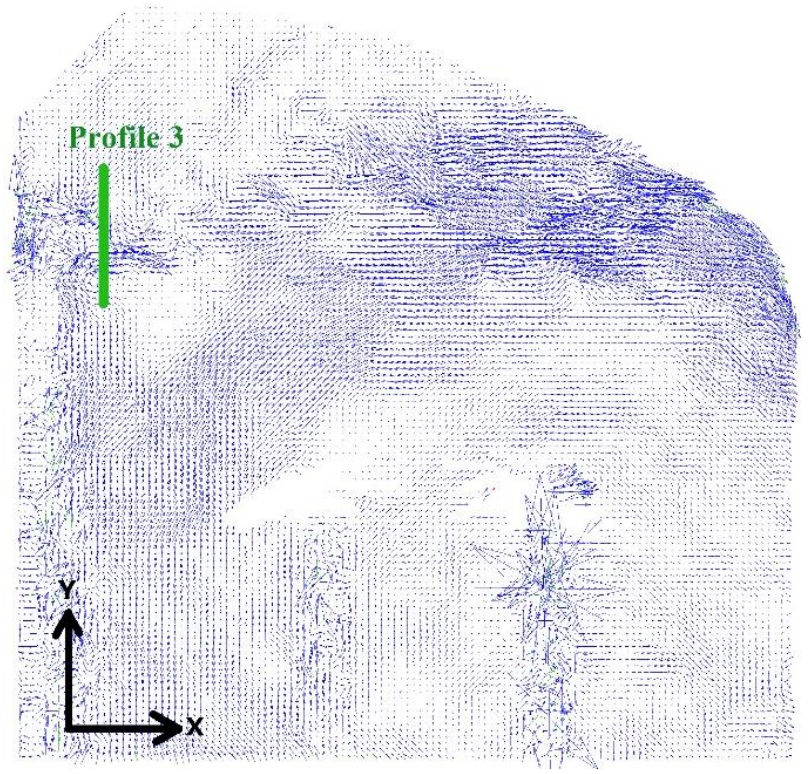

Fig. 10. Velocity vectors at measurement section S3, velocity profile marked Profile 3 , situated $3 \mathrm{~cm}$ in front of the diffuser.

Velocity profiles were extracted (Fig. 10) about $1 \mathrm{~cm}$ in front of the grid (in order to capture the merging jet phenomenon for section 1).

The results at grid measurement sections 1 and 2 show maximum velocity values around $0.2 \mathrm{~m} / \mathrm{s}$, while at the same time grid section 3 shows velocity values below 0.05 $\mathrm{m} / \mathrm{s}$. The values in the central area, being far greater than those expected and the small values in section 3 prompt further investigation.

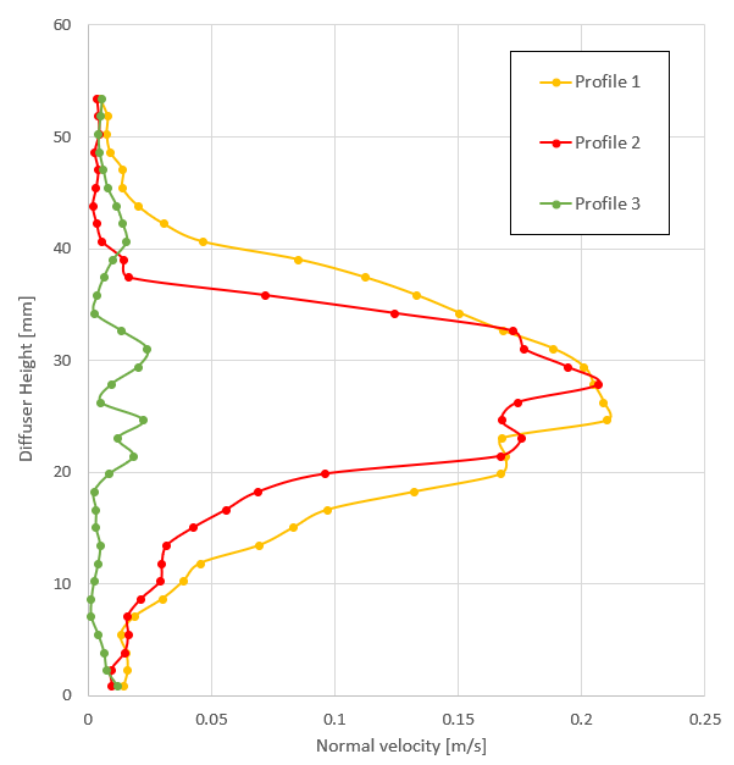

Fig. 11. Velocity profiles at $3 \mathrm{~cm}$ in front of the diffuser for each measurement section.

A flow visualization test was performed using a copper sulfate-based fluid. The visualization in Fig 11 shows the flow distribution in the plenum chamber upstream of the diffuser. Expected behavior was that the water would fill up the small chamber and flow out of the whole grid. Results proved different, the chamber was 
sufficiently small and the flow rate large enough that the water entering the chamber, instead of filling it up, hit the central part of the grid and entered the basin through a small fraction of the available surface. At the same time, this impact prompted recirculation areas to form left and right of the central grid wall, explaining the negative velocity values as being influenced by the recirculating flow.



Fig. 12. Flow visualization in the plenum chamber and at the jet exit.

This development prompts a recalculation of the average velocity values. Estimating that the flow rate passes through roughly 8 of the 26 columns (about a third) in the diffuser, for a flow rate of $71 / \mathrm{min}$ we have an average velocity value of $0.15 \mathrm{~m} / \mathrm{s}$. These new values are closer to what can be seen in the PIV measurements and could plausibly be accurate if the exact value of the open grid surface used would be known.

Other notable observations include a cone of light originating from a joint at the top of the basin. This highly illuminated area can affect measurement accuracy especially since it is present near the areas which are of most interest in the flow field. Due to the refraction of the light through this joint, a darker area is formed right near the diffuser. This phenomenon owes its presence to the structure of the model itself and cannot be remedied without seriously affecting measurement accuracy.

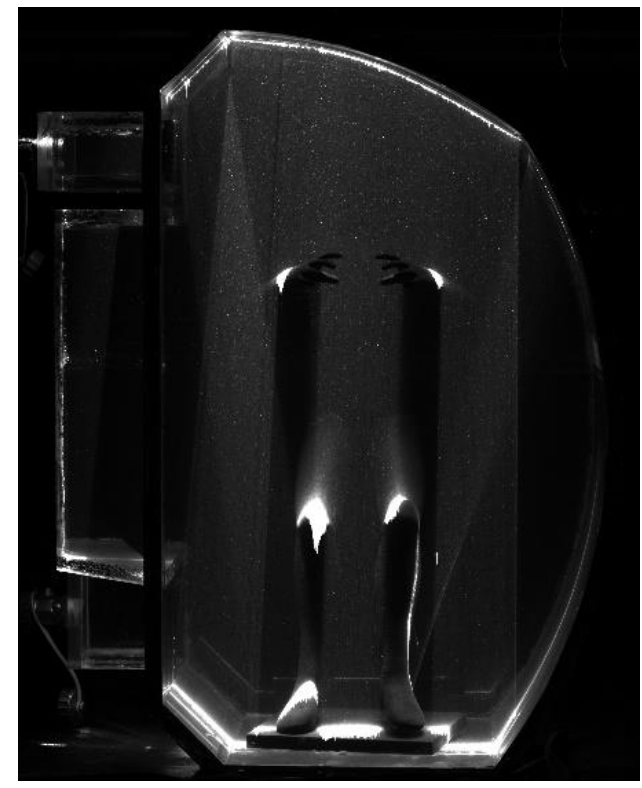

Fig. 13. Cone of light generated by wall joint.

\section{Conclusions and future improvement proposals}

Current results are not sufficient for the numerical validation intended in our initial research plan. The main reason is the distribution of the flow rate on the diffuser, which was initially assumed to be relatively evenly distributed, but has actually proved to be heavily biased towards the center going so far as to present unusual recirculation areas uphill of the grid. The experimental setup and the materials required have been verified during the 3 tests.

Impediments in the experimental procedures were almost entirely related to the build quality and design features of the small-scale model. Future research in this direction will require a solution to these issues. A second small-scale model is currently in the works featuring the following improvements:

- Several channels will be built leading up to the introduction grid, with the aim of evenly distributing the fluid flow through all grid sections, avoiding the central area bias and the recirculation areas. Several supply ducts will also increase the global flow rate.

- The joint present on the top side of the model will be removed and the whole top side will be replaced by a single continuous piece of acrylic. This more expensive solution will alleviate stress present at the joints and remove the cone of light which previously formed.

- An air purge will also be installed from the start in a corner of the top wall of the model. Larger diameters for water evacuation will be provided in order to compensate for the increased flow rate made possible by the multiple channels built at the entrance.

- Several structural improvements are envisioned in order to increase the pressure tolerance of the model.

The measurement methods and calibration techniques proved to be accurate when compared to accurate assumptions of fluid flow behavior. It is expected that the model improvements coupled with these measurement techniques will yield accurate results and offer at the end of the day useable results for numerical validation.

This work was supported by a grant of the Romanian space agency ROSA, QUEST - Advanced air diffusion system of the crew quarters for the ISS and deep space habitation systems, STAR-CDI-C3-2016-577 and by a scholarship from the University of Rennes 1.

\section{References}

[1] C. Son, J. Zapata, and C. Lin, "Investigation of Airflow and Accumulation of Carbon Dioxide in the Service Module Crew Quarters," Int. Conf. Environ. Syst., no. 01, p. 2341, 2002.

N. Sp--, "National Aeronautics and Space Administration HUMAN INTEGRATION DESIGN HANDBOOK," Spaceflight (Lond)., 
pp. 1-27, 2010.

[3] T. P. Schlesinger and B. R. Rodriguez, "International Space Station Crew Quarters OnOrbit Performance and Sustaining Activities," Int. Conf. Environ. Syst., pp. 1-9, 2013.

[4] C. M. Matty, "Overview of Carbon Dioxide Control Issues During International Space Station/Space Shuttle Joint Docked Operations," 40th Int. Conf. Environ. Syst., vol. 2, pp. 1-9, 2010.

[5] J. Broyan, D. Welsh, and S. Cady, "International Space Station Crew Quarters Ventilation and Acoustic Design Implementation," 40th Int. Conf. Environ. Syst., pp. 1-16, 2010.

[6] C. P. Coutinho, A. J. Baptista, and J. Dias Rodrigues, "Reduced scale models based on similitude theory: A review up to 2015," Eng. Struct., vol. 119, pp. 81-94, 2016.

[7] C. Croitoru, I. Nastase, A. Iatan, V. Iordache, and A. Meslem, "Numerical and experimental modeling of airflow and heat transfer of a human body," 2011.

[8] P. Danca, F. Bode, I. Nastase, and A. Meslem, "CFD simulation of a cabin thermal environment with and without human body - thermal comfort evaluation," E3S Web Conf., vol. 32, p. 01018, Feb. 2018. 